\title{
On some properties of hollow and hollow dimension modules
}

\author{
Majid Mohammed, Abd Ghafur Bin Ahmad \\ School of Mathematical Science, Faculty of Sciences and Technology, Universiti Kebangsaan Malaysia
}

Email address:

m_m_ukm@ymail.com(M. Mohammed),ghafur@ukm.my(A. G. B. Ahmad)

\section{To cite this article:}

Majid Mohammed, Abd Ghafur Bin Ahmad. On Some Properties of Hollow and Hollow Dimension Modules. Pure and Applied Mathematics Journal. Vol. 2, No. 5, 2013, pp. 156-161. doi: 10.11648/j.pamj.20130205.12

\begin{abstract}
No doubt, a notion of the hollow dimension modules can constitute a very important situation in the module theory. Therefore, our work presents a key role mainly in some properties and characterizations of hollow and hollow dimension module. We prove that if $\mathrm{R}$ be a V-ring and $\mathrm{M}$ is semisimple with indecomposable property, then $\mathrm{M}$ is hollow module. Also we study characterization the relation between lifting property and hollow-lifting module. We prove that if $\mathrm{M}$ is a nonzero indecomposable and lifting module over a commutative noetherian ring $\mathrm{R}$ then $\mathrm{M}$ is hollow module. Let $\mathrm{M}$ be an R-module and $\mathrm{N}$ be a submodule of $\mathrm{M}$ if $\operatorname{hdim}(\mathrm{M})=\operatorname{hdim}\left(\frac{M}{N}\right)+\mathrm{hdim}(\mathrm{N})$, then $\mathrm{M}$ is supplemented module.
\end{abstract}

Keywords: Hollow Module, Indecomposable Module, Hollow Dimension Module, Hollow-Lifting Module

\section{Definitions and Notations}

Throughout this paper, all rings will have identities and all modules will be unital right modules. Let $M$ be a module. Any small submodule $K$ of $M$ is denoted by $(K \ll M)$. A submodule $K$ of $M$ is small in $M$ if for every submodule $L$ of $M \ni K+L=M$ then $K=M$. A module $M$ is called lifting if, for all $N$ submodule of $M$, there is a decomposition $M=$ $H \oplus G \ni H$ submodule of $N$ and $(N \cap H) \ll M$. We call a non-zero $R$-module $M$ hollow if every proper submodule is superfluous in $M$. Or a module $H$ is said to be hollow if it is an indecomposable lifting module. Therefore we can say any factor modules of hollow modules are again hollow. If $M$ has a largest submodule, i.e. a proper submodule which contains all other proper submodules, then $M$ is called a local module therefore it is obvious that a largest submodule has to be equal to the radical of $M$ and in this case $\operatorname{Rad}(M) \leqslant M$. Let $M$ be an $R$-module and $N, L$ are submodules of $M$ then $L$ is a radical supplement ( $R \mathrm{ad}-$ supplement) of $N$ in $M$ if $N+L=M$ and $(N \cap L) \subseteq \operatorname{Rad}(L)$. Therefore $M$ is $R$ ad-supplemented if every submodule in $M$ has a Rad-supplement. A module $\mathrm{M}$ is called $\left(D_{1}\right)$-module if for every submodule $A$ of $M$, there is a decomposition $M=M_{1} \oplus M_{2}$ such that $M_{1} \leq A$ and $\left(M_{2} \cap A\right) \ll M$ and a module $M$ has $\left(D_{2}\right)$ property if $N \leq M \ni\left(\frac{M}{N}\right)$ is isomorphic to a direct summand of $M$, then $N$ is a direct summand of $M$.
A module $M$ is called $\left(D_{3}\right)$ if for every direct summands $K$ and $L$ of $M$ with $M=K+L$ and $(K \cap L)$ is a direct summand of $M$. Recall that a lifting module $M$ is called quasi-discrete module if $M=M_{1}+M_{2} \ni M_{1}, M_{2}$ are direct summands of $M$ and so $\left(M_{1} \cap M_{2}\right)$ also is a direct summand of $M$. Therefore if we have $M$ has $\left(D_{1}\right)$-property and $\left(D_{3}\right)$ property imples $M$ is quasi-discrete module. We recall that a ring $R$ is a right $V$-ring if and only if every simple $R$ module is injective, if and only if $\operatorname{Rad}(M)=0$. Let $N \leq M$ such that $N$ is proper and in a maximal submodule of $M$, then $M$ is coatomic module; or equivalently, let $N$ submodule of $M$, and radical of $\left(\frac{M}{N}\right)$ equal $\left(\frac{M}{N}\right)$ implies that $M$ equal $N$ and if every submodule of $M$ is a direct summand in $M$ then we can say that $\mathrm{M}$ is semisimple. See the following examples:

*) semisimple modules, are coatomic.

*) finitely generated modules, are coatomic module.

*) hollow modules and local modules are coatomic modules.

This paper is divided into four different sections. In section 2 we study the relations between hollow and indecomposable module and we prove that if $R$ be a $V$-ring and if $M$ is semisimple and indecomposable, then $M$ is hollow module. In section 3 we conclude some properties of hollow module and the relation between hollow and hollow-lifting module. Finally in section 4 we conclude some properties of hollow dimension and the relation 
between dimension property and hollow module.

\section{Hollow and Indecomposable Module}

In this section, we study hollow module and we give some properties to explaine the relation between indecomposable and hollow module. A module $M$ is called indecomposable if $M \neq 0$ and it is not a direct sum of two nonzero submodules. Let $R$ be a Dedekind domain. Then every torsion-free divisible left $R$-module is $R$ adsupplemented.

$\left(\mathrm{P}_{1}\right)$ Let $R$ be Neotherian ring. If $M$ lifting module and have indecomposable property, then $M$ is hollow module.

Also, an $R$-module $M$ is called c-f-lifting if every submodule of $M$ which contained coessentialy in a finitely generated submodule lies a bove a direct summand. Therefore if $M$ have $D_{1}$ property ( $M$ is lifting module) then it is c-f-lifting and so:

$\left(\mathrm{P}_{2}\right)$ For every coessentialy finitely generated submodule $N$ of $M \ni M_{1} \subseteq N,\left(N \cap M_{2}\right) \leqslant M$, then we can get a decomposition $M=M_{1} \oplus M_{2}$.

\section{Theorem 2.1.}

Let $R$ be a Dedekind domain and commutative noetherian ring and let $M$ satisfies the following statements:

1. $M$ is hereditary module with small radical.

2. $M$ is torsion-free divisible left $R$-module.

3. $M$ is indecomposable module.

Then $M$ is hollow module.

Proof: From condition 2 if $K$ be the field of quotient of $R$. Then radical of $K$ equal $K$ implies $K$ is $R$ ad-supplemented and since $M$ is a torsion-free divisible module, then it is a direct sum of copies of $K$. This means $M$ is a Radsupplemented module. Let $B$ any supplement submodule of $M$. Thus, $B$ is a direct summand of $M$. Also let $A$ be any submodule of $M$ and since $M$ is a $\operatorname{Rad}$-supplemented module, then there exists a submodule $B$ of $M$ such that $A+$ $B=M$ and $(A \cap B)$ subset of radical of $B$. Now $(A \cap B)$ subset of radical of $B$ and so subset of radical of $M$ and it small of $M$ therefore $(A \cap B)$ small in $M$ :

Thus $(A \cap B)$ small in $B$ and so $M$ is supplemented module. Hence $M$ is an amply supplemented module, and so is lifting and hence] $M$ is hollow module.

\section{Theorem 2.2.}

Let $R$ be a commutative noetherian ring and let $M=\oplus$ $N_{\mathrm{i}}, i=1, \ldots, n$, such that $M$ satisfy the following conditions:

1- $N_{1}, N_{2,}, . ., N_{\mathrm{n}}$, are hollow modules.

2- $M$ has $\left(D_{3}\right)$ property.

3- $M$ is quasi-discrete module.

4- $M$ is indecomposable module.

Then $M$ is hollow module.

\section{Theorem 2.3.}

Let $M_{1}$ and $M_{2}$ be hollow modules with local endomorphism rings. Assume that there is no epimorphism between $M_{1}$ and $M_{2}$ such that $M=M_{1} \oplus M_{2}$. If $M$ is quasidiscrete; then $M$ is hollow module.
Proof: By [ 10, Theorem 4.5 and Corollary 2.8].

\section{Lemma 2.4.}

[16, Lemma 3.16] Let $R$ be a $V$-ring. An $R$-module $M$ is lifting if and only if it is semisimple.

\section{Theorem 2.5.}

Let $R$ be a commutative noetherian ring and let $\mathrm{M}$ be an $R$-module satisfying the following:

1 - Every simple left $R$-module is injective.

$2-M$ is semisimple $R$-module.

3- $\mathrm{M}$ is indecomposable $\mathrm{R}$-module.

Then $M$ is hollow module.

Proof: Since every simple module $M$ over any $\operatorname{ring} R$ is injective then $R$ is $V$-ring, but $M$ is semisimple then by [ Lemma 2.4] $M$ is lifting module therefore for all $N$ submodule of $M$, there is a decomposition $M=H \oplus G \ni H$ $\leq N$ and $(N \cap H) \ll M$, but $R$ is commutative noetherian ring with indecomposable property implies $M$ is hollow module [Property $\mathrm{P}_{1}$ ].

\section{Theorem 2.6.}

Let $M$ be an $R$-module. If $M$ is local, then $M$ is hollow module.

Proof: By definition of local module we can say that every proper submodule $N$ of $M$ implies $N$ subset of radical of $M$ and radical of $M$ is small in $M$ (i.e. $M$ has a largest proper submodule). Then $N$ is small in $M$ and by definition of hollow module we get the proof.

\section{Theorem 2.7.}

Let $M$ be a nonzero module such that it satisfying the following:

$1-M \neq \operatorname{Rad}(M)$.

2- Every submodule of $M$ lies over a summand of $M$.

3 - $M$ is indecomposable module.

Then $M$ is hollow.

Proof: We must prove that $M$ is local module. Let $M$ be a nonzero module such that $\mathrm{M}$ satisfying conditions 2 and 3 . Let $N$ be a proper submodule of $M$. Then $M=L \oplus K, L$ submodule of $N$ and $(K \cap N)$ is small in $M$. Since $L \neq M$, $K=M$ and $N=(K \cap N) \sqsubset \operatorname{Rad}(M)$ then radical of $M$ is the unique maximal submodule of $M$. Thus $M$ is local and by [Theorem 2.6] $M$ is hollow module.

\section{Theorem 2.8.}

Let $M$ be an $R$-module. If $M$ is hollow module, then $M$ is supplemented module .

Proof: Let $M$ be an $R$-module and $K$ be a submodule of $M$. Then $K+M=M$. By hypothesis, $(K \cap M)=K \leqslant M$. Hence $M$ is supplemented module.

\section{Remark 2.9.}

If $M$ has no maximal submodule $(\operatorname{Rad}(M)=M)$, then all finitely generated submodules of $M$ are small in $M$ and this means $M$ be finitely supplemented but not hollow. 


\section{Theorem 2.10.}

Let $M$ be $\left(D_{1}\right)$ - module and let $M_{1}$ and $M_{2}$ are hollow modules, then $M$ is a direct summand of hollow modules.

Proof: Since $M$ is $\left(D_{1}\right)$ - module then $M$ is lifting module and so $M$ is c-f-lifting. Now by $\left(P_{2}\right)$ property $M$ is a direct summand of hollow modules $\left(M=M_{1} \oplus M_{2}\right)$

\section{Corollary 2.11.}

Let $R$ be a Neotherian and right perfect ring then every indecomposable projective right $R$-module is hollow.

\section{Corollary 2.12.}

Let $R$ be a ring such that it is right perfect commutative noetherian. If $M$ is indecomposable $R$-module then $M$ is hollow module.

\section{Corollary 2.13.}

Let $M$ be projective and indecomposable module such that every factor module of $M$ has a projective cover, then $M$ is hollow module.

\section{Corollary 2.14.}

Let $M$ be an $R$-module. If $M$ is hollow and radical of $M$ not equal $M(\operatorname{Rad}(M) \neq M)$, then $M$ is local.

\section{Hollow and Hollow-Lifting Module}

In this Section we conclude some properties to study the relation between hollow and hollow-lifting. There is an important question which is how we can use the indecomposablity with hollow-lifting to get lifting module? Let $M$ be a direct summand of hollow modules $H_{\mathrm{i}}, i=1, \ldots, \mathrm{n}$, and let $M$ is $\left(D_{3}\right)$-module, then a module $M$ is called hollow-lifting if every submodule $N$ of $M$ with $(M / N)$ hollow has a coessential submodule in $M$ that is a direct summand of $M$ then the following are equivalent:

(1) $M$ is hollow-lifting.

(2) $M$ is lifting.

(3) $M$ is quasi-discrete;

Let $M=\sum M_{\mathrm{i}}, \mathrm{i}=1, ., n$ such that all $M_{\mathrm{i}}$ are hollow module and let $M$ has $\left(D_{2}\right)$ property with hollow-lifting, then $M$ is lifting module. For an indecomposable module $M$, the module $M$ is hollow-lifting if and only if $M$ is hollow, or else $M$ has no hollow factor modules and also semisimple modules are hollow-lifting.

\section{Theorem 3.1.}

Let $M=\left(M_{1} \cap M_{2}\right)$ be an indecomposable module. If $M$ is hollow-lifting then it is hollow module.

Proof: Suppose that $\left(M_{1} \cap M_{2}\right)$ has a hollow factor module. Then there exists a proper submodule $N$ of $\left(M_{1} \cap\right.$ $\left.M_{2}\right) \ni M_{1} \cap\left(M_{2} / N\right)$ is hollow. Since $\left(M_{1} \cap M_{2}\right)$ is hollowlifting, then there exists $K$ a direct summand of $\left(M_{1} \cap M_{2}\right)$ $\ni(N / K) \ll M_{1} \cap\left(M_{2} / K\right)$ and since $\left(M_{1} \cap M_{2}\right)$ is indecomposable module then $\left(M_{1} \cap M_{2}\right)$ not equal zero and it is not a direct sum of two nonzero submodules and this means $K=0$ and $N$ small in $\left(M_{1} \cap M_{2}\right)$. Therefore $\left(M_{1}\right.$ $\left.\cap M_{2}\right)=M$ itself is a hollow module.

\section{Theorem 3.2.}

Let $M_{1}, M_{2}$ be modules having no hollow factor modules. Then $M=M_{1} \oplus M_{2}$ is hollow-lifting.

Proof: Suppose that $M$ has a submodule $N$ such that $\left(\frac{M}{N}\right)$ is hollow. Since $\frac{M 1+N}{N}+\frac{M 2+N}{N}=\frac{M}{N}$, there exists $i=1, \ldots, n$ $\exists \frac{M i+N}{N}=\frac{M}{N}$ is hollow. So $M_{\mathrm{i}}$ has a hollow factor module, but $M_{1}$ and $M_{2}$ are having no hollow factor modules (contradiction). Therefore $\left(M_{1}+M_{2}\right)$ is hollow-lifting.

\section{Example 3.3.}

Let $p$ be any prime integer then $\left(Z / p^{2} Z \oplus Z / p^{3} Z\right)$ is lifting, therefore it is hollow-lifting. Also $(Z / p Z) \oplus(Z /$ $\left.p^{3} Z\right)$ it is not lifting and this implies it is not hollow-lifting (see [13]).

\section{Proposition 3.4.}

Let $M=H_{1} \oplus H_{2} \oplus \ldots \oplus H_{\mathrm{n}}$ such that $H_{1}, \ldots, H_{\mathrm{n}}$ be hollow modules. Then if $M$ is hollow-lifting module then $M$ is lifting module.

Proof: Let $N \leq M$. If we have the projections $\pi_{1}: M \rightarrow H_{1}$, $\pi_{2}: M \rightarrow H_{2}, \ldots$, and $\pi_{\mathrm{n}}: M \rightarrow H_{\mathrm{n}}$. If $\pi_{1}(N) \neq H_{1}, \pi_{2}(N) \neq$ $H_{2}, \ldots$, and $\pi_{\mathrm{n}}(N) \neq H_{\mathrm{n}}$ then $N \ll M$. Suppose that $\pi_{1}(N)=$ $H_{1}$. Then $M=N+H_{2}$. Therefore, $(M / N)$ is hollow. Hence there exists a direct summand $K$ of $M \ni K \leq N$ and $(N / K)$ $\ll(M / K)$. Thus $M$ is lifting.

Let $M$ be an $R$-module. Then the direct summand of two hollow-lifting modules not hollow-lifting. For example, let $M$ be the $Z$-module $(Z / 2 Z) \oplus(Z / 8 Z)$. We know that ( $Z /$ $2 Z)$ and $(Z / 8 Z)$ are hollow-lifting but $M$ not hollow-lifting. Also, when $M=M_{1} \oplus M_{2}$ be duo module and if $M_{1}$ and $M_{2}$ are hollow-lifting, then $M$ is also hollow-lifting module.

\section{Theorem 3.5.}

Let $M$ be an $R$-module with small radical. Let $N$ be submodule of $M$ such that if $N+K=M$ and $N$ is minimal with respect to this property then $M$ is hollow-lifting.

Proof: Firstly, we must prove that $\operatorname{Rad}(M)=0$. Suppose $\operatorname{Rad}(M) \neq 0$, there exists an element $r$ belong to $\operatorname{Rad}(M)$. We have $R_{\mathrm{r}}$ supplement then $R_{\mathrm{r}}+H=M$ and $R_{\mathrm{r}} \cap H$ is small in $R_{\mathrm{r}} \ni H \leq M$. Since $r \in(M), R_{\mathrm{r}} \ll M$ and $H=M$ and hence $R r \ll R r$ but this impossible. Then $\operatorname{Rad}(M)=0$, also $M=$ $N+N_{1}$ and $\left(N \cap N_{1}\right) \ll N \ni N_{1} \leq M$, therefore $(N \cap$ $\left.N_{1}\right) \subseteq \operatorname{Rad}(M)=0$, then $\left(N \cap N_{1}\right)=0$ and hence $M=$ $N_{1} \oplus N$, then $M$ is semisimple module hence $M$ is hollowlifting.

\section{Corollary 3.6.}

Let $M$ be an $R$-module satisfying the following conditions:

1- $M$ coatomic module.

2- $M$ supplemented module.

3- If $N$ submodule of $M$ such that $N$ is supplement in $M$. Then $M$ is hollow-lifting. 


\section{Corolary 3.7.}

Let $M$ be an $R$-module. If $M$ satisfy the following conditions:

$1-M$ is local module.

2- $M$ is coatomic module.

3- $\operatorname{Rad}(M)=0$.

Then $M$ is hollow-lifting.

\section{Finite Hollow Dimension Property}

A module $M$ is called to have finite hollow dimension if $M$ does not contain an infinite coindependent family of submodule. The module $M$ is said to have finite dual Goldie dimension if every coindependent family of submodules of $M$ is finite. Not that a module $M$ with dual Goldie dimension 1 is said to be hollow, and a cyclic hollow module is said to be local [6]. Let $N \leq R$-module $M$. If $M$ has finite hollow dimension then $M$ is weakly supplemented, therefore an $R$-module $M$ with $\operatorname{Rad}(M)=0$ is weakly supplemented if and only if $M$ is semisimple, in this case $\operatorname{hdim}(M)=\operatorname{length}(M)$ holds. If $R$ is semilocal ring and $M$ be finitely generated then $M$ has finitely hollow dimension. Also if $M$ is artinian module then any submodule $N$ of $M$ is semiartinian and so finitely hollow dimension. Let $M$ be an $R$-module with finite hollow dimension then $M$ is hollow-lifting if and only if $M$ is lifting, and so $M$ is c-f-lifting module [16]. A $Z$-module has finite hollow dimension if and only if it is artinian. Since every artinian module is amply supplemented and so supplemented then Z-module $M=Z / p Z \oplus Z / p^{3} Z$ is an amply supplemented module, where $p$ is any prime integer. Therefore $Z$-module is an amply supplemented module.

\section{Definition 4.1.}

A module $M$ has hollow dimension $n$, if there exists a small epimorphism from $M$ to a direct sum of $n$ hollow modules.

Or: a module $M$ is said to have hollow dimension (or finite dual Goldi dimension) if there exists an exact sequence:

$g: M \rightarrow \bigoplus H_{\mathrm{i}}, \mathrm{i}=1, \ldots, n, \ni H_{\mathrm{i}}$ hollow and kernel of $\mathrm{g}$ small in $M$.

\section{Remarks 4.2.}

1. We called $n$ hollow dimension (dual Goldi dimension) of $M$ and we write $\operatorname{hdim}(M)=n$.

2. If $M=0$, then hollow dimension of $M=0$, but if $M$ does not have finite hollow dimension, then hollow dimension of $M=\infty$.

3. If we have descending chain $H_{1} \supseteq H_{2} \supseteq \ldots$ of submodules of $M$ there exists $i \exists H_{\mathrm{i}}$ lies above $H_{\mathrm{k}}$ in $M, \forall$ $k \geq i$.

4. Any module is hollow if and only if it has hollow dimension 1.

Recall that every artinian module is amply supplemented and so supplemented then $Z$-module $M=Z / p Z \oplus Z / p^{3} Z$ is an amply supplemented module, where $p$ is any prime integer. Therefore $Z$-module is an amply supplemented module.

\section{Proposition 4.3.}

Let $M$ be a Z-module. If $M$ has finite hollow dimension then it is artinian module.

Proof: Let $M$ has finite hollow dimension. We must prove that $M$ is a finite direct sum of hollow modules. Suppose that $t(M)$ be the torsion submodule of $M$. Then, if $t(M) \neq M$, then we have hollow dimension of $(M / t(M))=$ $\infty$ and this implies hollow dimension of $M=\infty$, but this contradiction. Therefore $M=t(M)$, i.e., $M$ is torsion module. By induction on $n=\operatorname{hdim}(M)$ we show that $M$ is a direct sum of $n$ hollow $Z$-modules. If $n=1$ then $M$ is hollow module. Let $n \in N$, all Z-modules such that hollow dimension of $M=k$, and $1 \leq k \leq n$, are a direct sum of $k$ hollow modules. Let $M$ be an abelian torsion group with hollow dimension of $M=n+1$. Therefore $M$ is not indecomposable module, since $M \simeq \mathrm{Z}_{\mathrm{pk}}$ and so of hollow dimension 1 then there exists a proper decomposition $M=$ $M_{1} \oplus M_{2}$, let $(n+1)=$ hollow dimension of $M=n_{1}+n_{2}$ such that $n_{1}=$ hollow dimension of $M_{1}$ not equal zero and $n_{2}=$ hollow dimension of $M_{2}$ not equal zero. Then, we use mathematical induction, to obtain $M_{1}$ and $M_{2}$ are direct sums of $n_{1}$ and $n_{2}$ hollow modules respectively. Thus $M$ is a direct sum of $(n+1)$ hollow modules. Therefore every $Z$ module $M$ with finite hollow dimension is a finite direct sum of hollow modules. Let $M$ be hollow and let $(M / t(M))$ is hollow and torsion free, then we have $M=t(M)$. Hence $M$ is an indecomposable torsion abelian group and so, is isomorphic to $Z_{\mathrm{pk}}$ for some prime $p$ and $1 \leq k \leq \infty$ then these summands is isomorphic to an artinian module of the form $\mathrm{Z}_{\mathrm{pk}}$ with $p$ a prime and $1 \leq k \leq \infty$. Hence $M$ is artinian module.

Since every artinian module is amply supplemented and so supplemented then Z-module $M=Z / p Z \oplus Z / p^{3} Z$ is an amply supplemented module, where $p$ is any prime integer. Therefore Z-module is an amply supplemented module.

\section{Theorem 4.4.}

Let $H=H_{1} \oplus H_{2} \oplus \ldots \oplus H_{\mathrm{n}}$, such that $H_{i}$ are submodules of $H$, then $\operatorname{hdim}(H)=\operatorname{hdim}\left(H_{1}\right)+\operatorname{hdim}\left(H_{2}\right)$ $+\ldots+\operatorname{hdim}\left(H_{\mathrm{n}}\right)$.

Proof: Since each $H_{\mathrm{i}}$ is a factor of $H, \operatorname{hdim}(H) \geq \operatorname{hdim}\left(H_{\mathrm{i}}\right)$ because: let $N$ and $K$ be submodules of the $R$-module $H$. If $\left\{P_{1} / N, \ldots, P_{\mathrm{k}} / N\right\}$ is a coindependent family of submodules of $(H / N)$ then $\left\{P_{1}, \ldots, P_{\mathrm{k}}\right\}$ is a coindependent family of submodules of $H$. Hence $\operatorname{hdim}(H / N) \leq \operatorname{hdim}(H)$ and if $\operatorname{hdim}\left(H_{\mathrm{i}}\right)=\infty$ for any direct summand $H_{\mathrm{i}}$, implies $\operatorname{hdim}(H)$ $=\infty$. Now for all $i \in\{1, \ldots, k\}, \operatorname{hdim}\left(H_{\mathrm{i}}\right)=n_{\mathrm{i}}$ less than $\infty$ then, for each $1 \leq \mathrm{i} \leq k$, there is a coindependent family $\left\{H_{\mathrm{i} 1}, \ldots, H_{\mathrm{ini}} \leq H_{\mathrm{i}}\right\} \ni \cap H_{\mathrm{j}, \mathrm{j}}=1, \ldots, n_{\mathrm{i}}$ small in $M_{\mathrm{i}}$ and $\left(H_{\mathrm{i}} /\right.$ $\left.H_{\mathrm{ij}}\right)$ is a hollow module $\forall j=1, \ldots, n_{\mathrm{i}}$. For each $i_{0}$ belong to $\{1 \ldots, k\}$ and $\mathrm{j}_{0}$ belong to $\left\{1, . ., n_{\mathrm{i} 0}\right\}$, now define $\mathrm{H}_{1 \mathrm{i} 0 \mathrm{j} 0}$ to be the submodule of $H=\bigoplus H_{\mathrm{i}}, \mathrm{i}=1 \ldots \mathrm{k}$, given by $\Theta A_{\mathrm{i}}$, $\mathrm{i}=1, \ldots, \mathrm{k}, \ni A_{\mathrm{i} 0}=H_{\mathrm{i} 0 \mathrm{j} 0}$ and $A_{\mathrm{j}}=H_{\mathrm{j}}$ for $\mathrm{j} \neq \mathrm{i}_{0}$. Then it is straightforward to show that $\left\{\mathrm{H}_{1 \mathrm{i} 0 \mathrm{j} 0}: 1 \leq \mathrm{i}_{0} \leq \mathrm{k}, 1 \leq \mathrm{j}_{0} \leq \mathrm{n}_{\mathrm{i} 0}\right\}$ is coindependent family of $\sum n i \mathrm{i}=1 \ldots \mathrm{k} \leq H$ whose 
intersection is small in $H$ and $\left(H / \mathrm{H}_{1 \mathrm{i} 0 \mathrm{j} 0}\right) \simeq\left(H_{\mathrm{i} 0} / H_{\mathrm{i} 0 \mathrm{j} 0}\right)$ is a hollow module $\forall \mathrm{i}_{0}, \mathrm{j}_{0}$. Consequently, $\operatorname{hdim}(H)=\sum n i$, $\mathrm{i}=1 \ldots \mathrm{k}$

The module $M$ is said to have finite dual Goldie dimension if there exists an epimorphism from $M$ to a finite direct sum of $n$ hollow factor modules with small kernel. In this case $n$ is the dual Goldie dimension of $M$ and we denote $n$ by $\operatorname{codim}(M)$. Let $d: R$-Mod $\rightarrow N \cup\{\infty\}$ a rank function $R$-Mod if for all $M, N \in R$-Mod $=0 \Leftrightarrow d(M)=0$ and $d(M \oplus N)=d(M)+d(N)$ holds. Note that if $d$ is a rank funcrion and $M$ a module with $d(M)=1$, then $M$ is indecomposable. Therefore, $\operatorname{dim}(M)$ and $\operatorname{codim}(M)$ are rank functions.

\section{Theorem 4.5.}

Let $M$ be a lifting right $R$-module. If $M$ has finite dual Goldie dimension, then $M$ is a direct sum of hollow modules.

Proof: Suppose $M$ a lifting module such that there exists a rank function $d$ and $d(M)=1$. Then $M$ is indecomposable and hence hollow. Assume now $n \geq 1$ and assume that for every lifting module $N$ such that there exists a rank function $d$ with $d(N)<n, N$ is direct sum of hollow modules. Let $M$ be a lifting module and $d$ a rank function with $d(N)=n$. Let $M$ is indecomposable, then it is hollow. Otherwise $M$ has a decomposition $N \oplus L \ni N$ and $L$ are nonzerod. Therefore since we have $d(M \oplus N)=d(M)+d(N)$ and $n=d(M)+$ $d(N)$ then $d(N)$ and $d(L)<n$ and $N, L$ are lifting modules and by hypothesis they are finite direct sums of hollows and so is $M$.

\section{Theorem 4.6.}

An $R$-module $M$ with $\operatorname{Rad}(M)=0$ is weakly supplemented if and only if $M$ is semisimple, and in this case hollow dimension of $M=$ length $(M)$.

\section{Theorem 4.7.}

Let $M$ be an $R$-module with finite hollow dimension and $N$ be a submodule of $M$. If hollow dimension of $M=$ hollow dimension of $(M / N)+$ hollow dimension of $N$, then $M$ is supplemented module.

Proof : Suppose that $\operatorname{hdim}(M)=$ hollow dimension of $(M$ $(N)+$ hollow dimension of $N$. We must prove $N$ is a supplement of $K$ in $M$ for all $N$ ana $K$ are submodules of $M$. Since $M$ has finite hollow dimension, then $M$ is a small cover of a finite direct sum of hollow modules. Since hollow modules are weakly supplemented, so too is this direct sum, for every submodule $N \subseteq M, M_{1}+\left(M_{2}+N\right)$ has a trivial weak supplement. Also $\left(M_{2}+N\right)$ also has a weak supplement in $M$. Also we get a weak supplement for $N$. Then $M$ is weakly supplemented by. Then $N$ has a weak supplement K. By assumption, $K+N=M$ and $(K \cap N) \ll$

$M$. Thus $\operatorname{hdim}(M)=\operatorname{hdim}(M /(K \cap N))=\operatorname{hdim}(K /(K \cap N)$ $\oplus N /(K \cap N))=\operatorname{hdim}(K /(K \cap N))+\operatorname{hdim}(N /(K \cap N))$, $=\operatorname{hdim}(M / N)+\operatorname{hdim}(M / K)$ and so we have $\operatorname{hdim}(M)=$ $\operatorname{hdim}(M / N)+\operatorname{hdim}(M / K)$. Thus, from our assumption,
$\operatorname{hdim}(N)=\operatorname{hdim}(M / K)=\operatorname{hdim}(N /(N \cap K))$ and, in particular, $\operatorname{hdim}(N)$ is finite. we get $(N \cap K) \ll N$ and so $N$ is a supplement of $K$ in $M$. Hence $M$ is supplemented module.

\section{Theorem 4.8.}

Let $M$ be an $R$-module having finite hollow dimension and let $K, L$ be submodules of $M$ with $M=K+L$. If $K$ and $L$ are supplements of each other in $M$ then $\operatorname{hdim}(M)=$ $\operatorname{hdim}(K)+\operatorname{hdim}(L)$.

Proof: suppose that $K$ and $L$ are supplements of each other in $M$. Since $K \cap L<<K, \operatorname{hdim}(K)=\operatorname{hdim}(K / K \cap L)$. If $M$ has finite hollow dimension, then for any submodule $N$ of $M, N<<M \Leftrightarrow \operatorname{hdim}(M)=\operatorname{hdim}(M / N)$. Also since $L$ is a supplement submodule of $M$, if $M$ has finite hollow dimension and $N$ a submodule of $M$, then $N$ is a supplement submodule of $M \Leftrightarrow \operatorname{hdim}(M)=\operatorname{hdim}(M / N)+\operatorname{hdim}(N)$. Hence $\operatorname{hdim}(M)=\operatorname{hdim}(M / L)+\operatorname{hdim}(L)$. Therefore $h(M)$ $=h(K)+h(L)$.

\section{Theorem 4.9.}

Let $M$ be an amply supplemented module $M$ with finite hollow dimension. If $M$ is hollow-lifting then it is lifting module.

Proof: Suppose that $M$ is hollow-lifting and let $K$ be a coclosed submodule of $M$. Since $M$ has finite hollow dimension, $(M / K)$ has finite hollow dimension by induction on hollow dimension of $(M / K)$. If hollow dimension of $(M / K)$ is 1 , then $K$ is a direct summand of $M$, since $M$ is hollow-lifting therefore we assume that hollow dimension of $(M / K)$ is $n$ and for every coclosed submodule $T$ of $M$ such that $(M / T)$ has hollow dimension less than $n$, then $T$ is a direct summand of $M$. Let $(H / K)$ be coclosed in $(M / K) \ni(M / K) /(H / K)$ is hollow. Therefore, $H$ is coclosed in $M$. Hence $M=H \oplus \mathrm{H}_{1}$ for some submodule $\mathrm{H}_{1}$ of $M$ as $M$ is hollow-lifting. Then $K=H \cap$ $\left(K \oplus \mathrm{H}_{1}\right)$ and $(M / K)=(H / K) \oplus\left(K \oplus \mathrm{H}_{1}\right) / K$. Therefore $\left(K \oplus \mathrm{H}_{1}\right) / K$ is coclosed in $(M / K)$. Also, by [5], $\left(K \oplus \mathrm{H}_{1}\right)$ is coclosed in $M$. Also by mathematical induction, we get $\left(K \oplus H_{1}\right)$ is a direct summand of $M$, and so $K$ is a direct summand of $M$. Therefore $K$ is a direct summand of $M$. Hence $M$ is lifting.

\section{Theorem 4.10.}

Let $M$ be a hollow-lifting module and let $M_{1}$ and $M_{2}$ are direct summands of $M$ such that $M=M_{1}+M_{2}$, then $\left(M_{1} \cap\right.$ $M_{2}$ ) is also a direct summand of $M$. If $M$ has finite hollow dimension, then $M$ is lifting and it is a finite direct sum of hollow modules.

Proof: Suppose $M$ is hollow-lifting module and if $M_{1}$ and $M_{2}$ are direct summands of $M \ni M=M_{1}+M_{2}$, then $\left(M_{1} \cap\right.$ $M_{2}$ ) is also a direct summand of $M$. We must prove that $M$ is a finite direct sum of hollow modules. If we use again Mathematical induction on $\operatorname{hdim}(M) . \operatorname{Suppose} \operatorname{hdim}(M)=1$, then by [Remark 4.2(4)] $M$ is hollow module. Suppose $r$ greater than 1 and suppose that for every hollow-lifting module $A$ with $\left(D_{3}\right) \ni \operatorname{hdim}(A)$ less than $r, A$ is a finite direct sum of hollow modules. Let $M$ be a hollow-lifting 
module with $\operatorname{hdim}(M)=r$. Suppose that $M$ is indecomposable. Since $M$ has finite hollow dimension, there exists a proper submodule $B$ of $M \ni(M / B)$ is hollow. As $M$ is hollow-lifting, there exists a direct summand $C$ of $M \ni C \leq B$ and $(B / C) \ll(M / C)$. Then $M$ is hollow, a contradiction. Therefore we can assume that $M$ is not indecomposable. So $M$ has a decomposition $M=A \oplus D$ with $A$ and $D$ are nonzero submodules of $M$. Since hdim( $(M)$ $=\operatorname{hdim}(A)+\operatorname{hdim}(D)$, implies $\operatorname{hdim}(A)$ and $\operatorname{hdim}(D)$ are less than r. Therefore, by [10], $A$ and $D$ are hollow-lifting modules. By hypothesis they are finite direct sum of hollow modules and so $M$ is lifting.

\section{Corollary 4.11.}

For any submodule $N$ of an $R$-module $M$, we have $\operatorname{hdim}(M / N) \leq \operatorname{hdim}(M)$.

\section{Corollary 4.12.}

For any submodule $N$ of $R$-module $M$, such that $N$ is small in $M$, then $\operatorname{hdim}(M)=\operatorname{hdim}(M / N)$.

\section{Corollary 4.13.}

Let $M$ be an $R$-module and $N, L$ submodules of $M$. Then $\operatorname{hdim}(N \oplus L)=\operatorname{hdim}(N)+\operatorname{hdim}(L)$.

\section{Corollary 4.14.}

Let $M$ be an $R$-module. If $M$ has finite hollow dimension; then $M$ is semilocal.

\section{Corollary 4.15.}

Let $A$ be a Z-module. If $A$ is nonzero and torsion free then $\operatorname{hdim}(A)=\infty$.

\section{References}

[1] A. Idelhadj, R. Tribak, On Some Properties of $\oplus$ Supplemented Modules, IJMMS :69, 4373-4387, 2003.

[2] C. Lomp, On Dual Goldie Dimension, Diplomarbeit (M. Sc. Thesis), Doesseldorf (1996).
[3] C. Lomp, On semilocal modules and rings, Comm. Algebra 27(4), (1999), PP. 1921-1935.

[4] D. Keskinl and C. Lomp, On Lifting LE-Modules, Vietnam Journal ol Mathematics 3O:2, 167-176, 2002.

[5] D. Keskin, On lifting modules, Comm. Algebra, 28(7), 3427-3440, 2000.

[6] D. Herbera and A. Shamsuddin, Moduleswith Semi-local Endomorphism Ring, proceedings of the american mathematical society, Vol 123, No 12, 1995.

[7] D. K. Tutuncu and S. H. Mohamed, Weak Lifting Modules with Small Radical, Ring and Module Theory Trends in Mathematics, 129-134, 2010 Springer Basel AG.

[8] E. Turkmen and A. Pancar, On Radical Supplemented Modules, International Journal of Computational Cognition, Vol. 7, No. 1, MARCH 2009.

[9] J. Clark, C. Lomp, N. Vajana, and R. Wisbauer. Lifting Modules. Birkhauser Verlag Basel, Boston-Berlin, 2006. $22: 3$

[10] N. Orhan, D. K.Tutuncu and R.Tribak, On Hollow-Lifting Modules, Taiwanese Journal of Mathematics, Vol. 11, No. 2, 545-568, June 2007.

[11] O. Nil, K. T. Derya, Hollow dimension of modules, J Zhejiang Univ SCI 2, 6A(10):1055-1057, 2005.

[12] R. Wisbauer, Foundations of Module and Ring Theory, Gordon and Breach, Reading, Philadelphia, (1991).

[13] S. H. Mohamed and B. J. Muller, Continuous and discrete modules, London Math. Soc. LNS 147 Cambridge Univ. Press, Cambridge, 1990., Proposition A.7

[14] S. Top, Totally weak Supplemented Modules, Thesis Master, July (2007), Izmir.

[15] T. Amoozegar, On Generalized Lifting Modules,Tarbiat Moallem University, 20th Seminar on Algebra,2-3 Ordibehesht, 1388 (Apr. 22-23, 2009) pp 21-23.

[16] Y. Talebi, A Generalization of Lifting Modules, Int. J. Contemp. Math. Sciences, Vol. 2, no. 22, 1069 - 1075, 2007. 